\title{
Recombinant viruses as vaccines against viral diseases
}

A.P.D. Souza ${ }^{1}$,

L. Haut ${ }^{1}$,

A. Reyes-Sandoval ${ }^{2}$ and A.R. Pinto ${ }^{1}$

\author{
1Departamento de Microbiologia e Parasitologia, \\ Universidade Federal de Santa Catarina, Florianópolis, SC, Brasil \\ ${ }^{2}$ Wellcome Trust Centre for Human Genetics, University of Oxford, \\ Roosevelt Drive, Oxford, UK
}

\section{Correspondence}

A.R. Pinto

Departamento de Microbiologia e

Parasitologia, UFSC

88040-900 Florianópolis, SC

Brasil

Fax: +55-48-331-9258

E-mail: pintoar@ccb.ufsc.br

A.R. Pinto is supported by Fundação de Ciência e Tecnologia do Estado de Santa Catarina (FUNCITEC) and

Programa Nacional de DST/AIDS,

Ministério da Saúde, Brasil.

Received August 30, 2004 Accepted February 21, 2005

\begin{abstract}
Vaccine approaches to infectious diseases are widely applied and appreciated. Amongst them, vectors based on recombinant viruses have shown great promise and play an important role in the development of new vaccines. Many viruses have been investigated for their ability to express proteins from foreign pathogens and induce specific immunological responses against these antigens in vivo. Generally, gene-based vaccines can stimulate potent humoral and cellular immune responses and viral vectors might be an effective strategy for both the delivery of antigen-encoding genes and the facilitation and enhancement of antigen presentation. In order to be utilized as a vaccine carrier, the ideal viral vector should be safe and enable efficient presentation of required pathogen-specific antigens to the immune system. It should also exhibit low intrinsic immunogenicity to allow for its re-administration in order to boost relevant specific immune responses. Furthermore, the vector system must meet criteria that enable its production on a large-scale basis. Several viral vaccine vectors have thus emerged to date, all of them having relative advantages and limits depending on the proposed application, and thus far none of them have proven to be ideal vaccine carriers. In this review we describe the potential, as well as some of the foreseeable obstacles associated with viral vaccine vectors and their use in preventive medicine.
\end{abstract}

\section{Introduction}

Vaccination has been one of the most important interventions designed to prevent disease to be employed on a worldwide basis, second only to the improvement of sanitation services and the provision of clean drinking water. The concept of vaccinology originated in the 18th century when the English physician Edward Jenner used cowpox virus inoculations in an attempt to combat
Key words - Vaccine

- Immunity

- Recombinant viruses

- Viral vectors

- Infectious disease smallpox infections. Since then, smallpox has been eradicated worldwide and other diseases such as poliomyelitis have virtually disappeared in countries of the Western Hemisphere and it is expected that some of these diseases will become globally eradicated in the near future. Also, vaccination has effectively limited the spread of many childhood and adult diseases, including measles, mumps, rubella, and hepatitis B. However, efforts to develop vaccines for remaining or 
emerging infectious agents, like malaria, AIDS, herpes, dengue fever, and some viral hepatitis have been futile. Despite considerable efforts, no licensed vaccines have yet been produced against any parasitic or fungal human disease. These difficulties are caused by the failure to clearly define antigen targets that induce specific and broad combinations of cellular, humoral and mucosal immune responses that can prevent infection and limit progression of the disease, without the risk of using live, attenuated vaccines. Consequently, new technologies are required to develop better and safer vaccines.

Novel approaches require a much more detailed characterization of the genetic organization of the pathogen and the genes responsible for virulence or induction of immunity. As a result of this increased knowledge and ability to manipulate the genomes of pathogens, we are in an unprecedented position to develop better live vectored vaccines that are safe and able to induce a wide range of protective immune responses including humoral and cellular immunity, as well as systemic and mucosal immunity. Presently, two approaches have been widely investigated: DNA vaccines and recombinant viruses. DNA vaccines have been extensively reviewed elsewhere (1). Recombinant viruses represent a particularly promising avenue of vaccine research, both for improving existing vaccines and for developing new ones. This process normally involves taking a proven safe and efficacious vaccine virus, such as vaccinia or adenovirus, and modifying its genome to include genes coding for immunogenic proteins from other pathogens. Virus-derived vectors offer several potential advantages over traditional vaccine technologies. These include, most notably, high-level production of protein antigens directly within cells of the immunized host, potential adjuvant effects of the viral delivery system itself and the possibility of efficient delivery of antigen directly to components of the immune system, such as antigen-presenting cells. A wide range of viruses have been extensively studied for their potential as recombinant vaccines and although each of them has its own unique characteristics, they all have the ability to induce not just humoral, but also cellular immune responses.

This review provides details on three viral vaccine vector systems (poxvirus, adenovirus, and alphavirus) receiving considerable attention in the literature and considered by the authors as the most advanced. In addition, two other promising viral vectors (poliovirus and herpesvirus), still in the initial stages of their development for use as vaccine vectors, will be discussed briefly. Some disadvantages of these systems will also be discussed, and some potential solutions on how to circumvent these shortcomings are offered.

\section{Poxvirus}

Members of the Poxviridae family are the most commonly used and best characterized viral vectors for vaccine purposes. The success of the World Health Organization (WHO)'s smallpox eradication program through the use of vaccinia virus, along with the development of genetic manipulation techniques for these viruses, have led to the development of poxviruses as genetic vectors for genes from many pathogenic organisms. These vectors were developed in the early eighties by cloning a foreign DNA into the genome of vaccinia virus, and the potential of recombinant vaccinia viruses as vaccine vectors was recognized immediately after their development (2).

Poxviruses are large viruses containing complex DNA genomes ranging in size between 130 to $300 \mathrm{~kb}$ pairs with a hairpin loop at each end (3). Although they are DNA viruses, their replication occurs in the cytoplasm of the infected cell due to the presence of a transcription machinery in the virions. 
The family Poxviridae is divided into two subfamilies, one of which, Chordopoxviridae, comprises the poxviruses of vertebrates. Chordopoxviridae consists of eight genera that are able to infect vertebrates; the genera Orthopoxvirus and Avipoxvirus have been widely engineered to be used as recombinant vectors for vaccine purposes. Variola virus, the causative agent of smallpox, and vaccinia virus, used in the smallpox vaccine, belong to the genus Orthopoxvirus and fowlpox and canarypox are two viruses belonging to the genus Avipoxvirus.

Certain characteristics make poxviruses suitable for use as vaccine vectors. Importantly, these vectors are very stable and, when lyophilized, they can be refrigerated and used even after 2 months. They are easy to manufacture at a low cost and, furthermore, have the potential to be administered by different routes, as shown by their safe delivery by the intradermal, intranasal, intravaginal, and intrarectal routes to generate antibody and T-cell responses. Oral administration of vaccinia recombinants has been shown to generate both mucosal and systemic immune responses to the recombinant protein. Finally, preexisting immunity to vaccinia virus, one of the main disadvantages of recombinant-viral vaccines, can be overcome by mucosal vaccination with vaccinia vectors due to compartmentalization of systemic and mucosal immune systems. Vaccinia induces a long-lasting immunity after a single immunization consisting of both humoral and cellular immune responses. Last but not least, one of the main features of this vector that gives it a tremendous advantage over other viral vectors is that large inserts of foreign DNA can be cloned into it, which allows for the insertion of multiple genes and the creation of multivalent vaccines. In a comparative study of several recombinant vectors for HIV vaccines, vaccinia vectors containing more than $10 \mathrm{~kb}$ of transgenic DNA have been used in trials, whereas other vectors such as adenoviral vectors can only accept less than $5 \mathrm{~kb}$ of genetic material (4). The possibility of introducing large amounts of DNA without disrupting viral stability allows for the insertion of several antigens even from different pathogens in the same recombinant vector, as has been shown with the insertion of the hepatitis B virus surface antigen, the herpes simplex virus glycoprotein $\mathrm{D}$ and the influenza virus hemagglutinin in the same vaccinia vector that generated an immune response to all three transgenic proteins upon injection in rabbits (5).

Recombinant technology utilizing vaccinia virus began around the time that the WHO declared smallpox eradicated in 1980 and recommended that the use of vaccinia virus for vaccine purposes be discontinued. Nevertheless, recombinant vaccinia vectors became an attractive tool for the study of the molecular biology of poxvirus for in vitro production of proteins and as a recombinant vaccine. The most useful strategy to make recombinant vaccinia vectors has been homologous DNA recombination within infected cells, a process that occurs naturally during viral replication. The use of plasmid vectors for DNA recombination which contain the complete expression cassette, including a promoter flanked by additional poxvirus sequences that direct recombination within a specified region of the genome, has facilitated the construction of vaccinia recombinants. The thymidine kinase (TK) gene has been useful to screen for viral recombinants, since new transgenes can be inserted into restriction sites flanking the TK promoter causing disruption of TK gene expression. After transfection with these plasmids, recombination occurs and viral vectors can be selected by the $\mathrm{TK}^{-}$phenotype in cells that are screened later for the expression of the transgene (6). Poxvirus vectors can also be created by direct ligation of genes into selected restriction sites within the viral genome, and transfection of the DNA into cells infected with a helper virus (7).

Some years ago, a rabies vaccine based 
upon recombinant vaccinia was developed and used to vaccinate foxes in the wild. This recombinant vaccinia vector-based vaccine was made by introducing the rabies glycoprotein cDNA into the TK gene and proved to be innocuous in different animal species and conferred protection by the oral route. This approach has allowed the administration of the vaccine through baits in largescale campaigns for the vaccination of wild life, dramatically reducing the incidence of rabies in previously prevalent areas (8). Although vaccinia-based vaccines have many advantages, their complex structure and the potential side effects caused by the vaccine carrier may make them unsuitable for widespread human use. Furthermore, as vaccinia can spread from the vaccinees to their immediate contacts, unintentional infection of immunocompromised individuals is of particular concern, especially in countries where HIV is endemic and a large pool of the population might be asymptomatic albeit HIV infected. Therefore, attenuated strains of vaccinia such as modified vaccinia Ankara (MVA) and NYVAC have become more appealing as candidates for vaccines due to their safety. MVA is a highly attenuated and well-characterized vaccinia virus derived from the Ankara strain by 570 serial passages in primary chick embryo fibroblasts, and has extensive genome deletions that make its growth very restricted, replicating only in chick embryo fibroblast cells (9). These modifications have helped to overcome many safety concerns and MVA is actively being tested as a vector in humans. A genetically engineered deletion mutant (strain NYVAC), derived from the Copenhagen strain of vaccinia virus has also been developed (10). This virus has deletions of 18 genes suspected of coding for vaccinia virus virulence factors. MVA and NYVAC-derived poxvirus vectors have been tested extensively in several animal models, as well as in humans, demonstrating their safety and immunogenicity $(11,12)$. Despite their success, these stud- ies raised concerns about the use of vaccinia vectors in individuals with preexisting immunity. In addition, a significant portion of the human population has received vaccinia virus as part of the smallpox eradication program and further vaccinations with other poxviruses may be ineffective in these people. In this regard, antigen-specific immune responses tend to be more consistent in vaccinia-naive vaccinees. Also, vector-specific immunity may potentially limit the effectiveness of multiple vaccinia vector administrations.

Therefore, interest has turned to avipox viruses, such as canarypox virus (CPV) and fowlpox virus (FPV), since these viruses do not replicate in human cells. Avipoxviruses initiate an abortive infection in non-avian cells, thus permitting expression of genes under the control of relevant viral promoters, without subsequent viral DNA replication and production of progeny virus (13). Furthermore, these vectors elicit less of a vector-specific immune response compared to attenuated vaccinia strains and thus can also be used for boosting several times. Avipoxvirus vector development was initially performed using FPV, an attenuated poultry vaccine. FPV recombinant viruses have been developed against several avian diseases and against animal rabies (14). Subsequently, CPV vectors were developed and proved more efficacious in mammals for reasons not entirely understood and vaccine vectors against cytomegalovirus, rabies and measles have been developed. CPV vectors have been tested in non-human primates and in human clinical studies $(10,11,15)$. A commercial version of canarypox vector, ALVAC, derived from the KANAPOX strain has also been developed (10). These ALVAC vectors do not induce cross-reactive immune responses against vaccinia-based vectors and therefore their immunogenicity is not compromised in vaccinia pre-exposed individuals. However, whether avipox-based vaccine vectors induce stronger cell-mediated 
immunity than orthopox vectors remains to be determined. Data from a few studies conducted to directly compare these two viral vectors suggest that vaccinia-based vectors are more potent $(11,15)$.

\section{Adenovirus}

Adenoviruses (Ad) are a group of viruses that belong to the Adenoviridae family. The popularity of Ad as a recombinant viral vector is largely due to the successful and safe immunization of millions of US military recruits with live enteric-coated capsules containing unattenuated Ad serotypes 4 and 7 in an attempt to prevent outbreaks of respiratory diseases caused by Ad. The safety of these vaccinations has been well documented (16). So far, no significant side effects or illnesses related to the use of these vaccines have been reported.

Ad vectors have several advantages over many conventional vaccines (17). They are relatively stable and easy to manipulate, can be readily obtained in high titers $\left(>10^{9}\right.$ plaqueforming units per $\mathrm{ml}$ ) by propagation on 293 or PER.C6 cells, and can be easily purified. Upon lyophilization the virus does not require refrigeration, which facilitates vaccine delivery especially in developing countries where cold chains are difficult to maintain. Ad vectors can be administered by various routes such as intranasal, oral, intratracheal, intraperitoneal, intravenous, intramuscular, or subcutaneous ones. Consequently, Adbased vaccines are able to induce not only a systemic immune response, but also, upon application to the respiratory or intestinal tract, a good mucosal immune response. Mucosal immune responses may be particularly useful to prevent infections by agents that infect the host via mucosal membranes. Importantly, the Ad genome has been shown to persist episomally rather than to integrate into its host DNA. This minimizes the risk of insertional oncogenesis and cellular gene activation. In general, there is usually no need to co-administer adjuvants to achieve a good immune response to the vaccine when immunizing with Ad-based vaccines and the vast majority of studies in animal models reported in the literature were conducted utilizing recombinant Ad (rAd) applied in saline.

Both replication-competent and replication-defective rAd have been in use for many years, either as experimental vaccines or as therapeutic agents for gene therapy (17). The most widely used cloning site in Ad is the E1 region. Since the gene products from the E1 region are critical for initiation of virus replication, deletion of this gene results in replication-defective virions, which renders them safe for in vivo application (18). In order to construct viable rAd that can carry heterologous DNA fragments, the function of replaced viral DNA has to be either dispensable or compensated for through a trans-acting source that can come from a helper virus, a helper cell line referred to as a packaging cell line, or both. The use of the packaging cell line is the most common and safest method of choice. Although the virus can be produced in E1expressing cell lines, once it is introduced into an animal that does not produce this gene product the viral infection results in an abortive infection (18). Another region of Ad suitable for foreign DNA insertion is the site of the E3 gene. E3 is not required for virus replication, so replacement of this region does not result in a replication-defective virus. Yet another region of the Ad genome suitable for cloning purposes is located at the right end of the viral genome in the $\mathrm{E} 4$ region. Combined deletions in E1, E3 and E4 can also be used, thus increasing the cloning capacity of the virus. However, the effects of multiple gene insertions into Ad vectors should be carefully assessed because these combinations could result in an overall change in the immune response or in its kinetics. The methods used to construct rAd have been reviewed in detail by other re- 
searchers $(18,19)$. To derive rAd vector particles, an antigen-expression cassette is first engineered into an Ad shuttle plasmid. This construct is then co-transfected along with the E1/E3-deleted Ad vector backbone into 293 cells, a human embryonic kidney cell line that stably expresses the Ad E1 region of the Ad viral vector (20). The antigen expression cassette is incorporated into the E1 region of the Ad vector by homologous recombination. This process is facilitated through site-specific recombination using bacteriophage or yeast recombinase and including their target sequences on the shuttle plasmid and vector (21). Propagation of the replication-incompetent Ad vector is also carried out in 293 cells. However, there is a risk of replication-competent Ad particles arising during production of replication-defective vector particles because of sequence complementarity between the vector and the Ad sequence found in 293 cells. This problem has been resolved by the development of an analogous E1-transformed human retinal cell line (PER.C6), which lacks these flanking sequences and is thus more suited for generation of vaccine stocks devoid of wildtype virus contaminants (22).

rAd have been shown to elicit strong humoral and cellular responses to foreign genes in all animal species tested so far, including primates, cattle, dogs, pigs, ferrets, hamsters, mice, rats, and rabbits. Genes from several important human and animal pathogens such as HSV, HBV, HPV, measles virus, HIV, SIV, and rabies have all been successfully expressed (17). Moreover, these immune responses have been shown to protect against disease in several animal models. The immune mechanisms by which protection was achieved have been investigated. Different routes of administration have been tested and were shown to influence the type of immune response elicited against the transgene product. Two of the most promising reports in recent years were studies on Ebola virus and HIV performed in non-human pri- mate models $(23,24)$. In both studies, immunized animals were resistant to challenge with lethal doses of virus.

In spite of the very encouraging results obtained in several animal models, and a limited number of clinical trials, there are several potential disadvantages that have to be considered in using Ad-based vectors. Safety is one of the most important issues that need to be considered. Vectors that are replication-deficient are preferable to those that are replication-competent. The potential oncogenicity and pathogenicity of some $\mathrm{Ad}$ (Ad4, Ad7, Ad11, Ad21, Ad37) especially in infants and immunocompromised subjects may call into question the safety of rAd vaccines based upon these serotypes as vaccine carriers for general use. Because some Ad (e.g., Ad12) can induce tumors in experimental animals, a search for human cancers induced by Ad was conducted. So far, Ad has not been shown to be associated with tumor development in humans. Another limitation of the use of Ad as a vaccine vector is the fact that Ad are ubiquitous in humans and nearly all adults possess antibodies to the common serotypes of human Ad. In the US, it has been shown that $35-45 \%$ of human adults have high titers of virus-neutralizing antibodies to human Ad of serotype 5 (25). A similar percentage of the population have neutralizing antibodies to human Ad4 or Ad7 (25). The percentage of pre-exposed humans may be higher in less developed countries or in a predominantly young adult population. Consequently vaccination in such populations may be ineffective as preexisting antibodies may eliminate recombinant virus particles before they are able to elicit an immune response. This approach was demonstrated in gene therapy trials when patients with pre-existing immunity to the vaccine carrier failed to develop antibodies to the transgene product upon application of the rAd vaccine (26). Similar results were obtained in experimental animals exposed to Ad prior to vaccination with a construct 
based on the homologous serotype (27). rAd from other species were shown to circumvent vector-specific neutralizing antibodies and the use of such constructs is being explored now. Some alternative adenoviral vaccine carriers are based on viruses that have been isolated from the lymphoid organs of chimpanzees (25) and their immunogenicity is not strongly impaired by preexisting immunity to common human serotypes of $\operatorname{Ad}(28,29)$. Moreover, the development of adenoviral vectors isolated from chimpanzees can not only overcome the preexisting immunity to human serotypes, but also allow for the sequential use of different serotypes in order to boost the immune response. This approach has been illustrated in mice and non-human primates using a combination of human and simian serotypes of Adbased vaccines in order to induce unprecedented immune responses (30).

Finally, adverse inflammatory responses have been reported in both animal models and human trials of adenovirus-based gene therapy vectors. These inflammatory responses may have caused the death of at least one subject in a gene therapy trial and have dramatically reduced the popularity of these virus vaccine vectors for human use. However, restricting the use of these vectors to vaccination and adapting them for oral administration could overcome this potential drawback. Another issue that may restrict the use of Ad-based vaccines is the host immune response against Ad proteins. The high immunogenicity of Ad proteins may prevent the repeated use of the same vaccine carrier. Strategies involving priming with a recombinant viral vector or naked DNA, followed by boosting with the recombinant protein or another viral vector have been used to circumvent the problems associated with the vector-specific immunity.

\section{Alphaviruses}

The use of alphaviruses as vaccine carri- ers has recently increased in popularity, but when compared with adenovirus or poxvirus systems these vaccine vectors are still in the initial stages of development. Alphaviruses are members of the Togaviridae family, which includes the human pathogens Western and Eastern equine encephalitis viruses. Two members of this group, Sindbis virus (SIN) and Semliki Forest virus, are not associated with any human disease, and together with Venezuelan equine encephalitis virus (VEE) are now being developed into expression vectors for vaccine applications $(31,32)$. Due to their simplicity and their many common features, there are no significant differences among them in their capacity to transduce host cells and express heterologous genes. Alphaviruses contain a single-stranded positive-sense RNA genome, which is approximately $12 \mathrm{~kb}$ in length, capped, polyadenylated and divided into two open reading frames (ORFs). The first ORF encodes the nonstructural proteins that are translated upon entry of the virus into the cytoplasm and are responsible for transcription and replication of viral RNA. The second ORF is under the control of a subgenomic promoter and normally encodes the structural proteins, which are responsible for encapsidation of viral RNA and final assembly into enveloped particles. Alphaviruses are very interesting vectors because they can infect a large number of animal cell types and express transient but high amounts of viral and heterologous proteins. Their replication occurs exclusively within the host cell cytoplasm, and thus provides inherent safety advantages for vectors derived from these viruses by eliminating any possibility of DNA integration events $(31,32)$. Furthermore, alphavirus vector-containing cells can eventually undergo apoptosis-dependent cell death and the release of apoptotic bodies that are efficiently taken up by antigen-presenting cells can result in enhanced immune cross-priming events (33). These features and the overall lack of preexisting immunity against alphaviruses in the 
human population underscore their potential as vaccine vehicles.

There are several possibilities for using alphavirus vectors in vaccine applications. Live attenuated alphaviruses have been used as models for producing vaccines (34). Recently, recombinant alphavirus-based vectors were created by replacing the viral structural protein gene with heterologous genes. These RNA vectors, known as replicons, are capable of replicating on their own but are not packaged into virus-like particles unless the structural proteins are provided in trans. Thus, replicons are single cycle vectors incapable of spreading from infected to noninfected cells and these particles cannot produce viral progeny because the viral structural genes are not packaged. Genetic manipulation of the replicon, including insertion of the foreign genes, is performed using a cDNA copy contained within a plasmid that is typically linked to a bacteriophage promoter enabling in vitro transcription of infectious vector RNA (35). The packaging of replicon RNA into particles can be accomplished by co-transfection of permissive cells with in vitro transcribed replicon RNA and helper RNA encoding the structural proteins under the control of their native subgenomic promoter. Helper RNAs maintain the 5 '- and 3'-end cis signals for co-amplification with the replicon, but are devoid of any replicase genes and packaging signals (35). However, a low frequency of replicationproficient particles may be produced through recombination between the expression and helper vectors. This problem has been addressed by engineering a two-helper system in which the capsid and membrane proteins are located on separate plasmids, thus reducing the likelihood of homologous recombination to negligible levels (36). Recently, stable alphavirus replicon particle packaging cell lines derived from BHK cells have been established (37). These cell lines contain two integrated DNA cassettes, encoding the capsid or envelope glycoprotein genes in a split helper configuration similar to the transient system. These cell lines will be invaluable for scientific studies as well as for large-scale production of alphavirus vectors.

In addition to using recombinant alphavirus particles, nucleic acids containing the alphavirus replicon can be directly administered to a host. The key component of the inoculation is the replicon (i.e., the nonstructural alphavirus genes), responsible for the rapid generation of some 200,000 RNA copies per host cell through direct RNA replication in the cytoplasm (38) and either DNA or RNA may be employed. The naked RNA approach consists of direct administration of in vitro transcribed RNA molecules containing the alphavirus nonstructural genes and the therapeutic gene of interest as a means of generating an immune response. The alternate approach is to use DNA-layered alphavirus vectors which utilize eukaryotic RNA polymerase II type promoters to drive the transcription of self-amplifying replicon vectors (39). The advantage of this modality is the simplicity of direct administration of plasmid DNA. Another advantage of administering nucleic acid molecules containing alphavirus replicons in comparison with other conventional nucleic acid-based vaccination regimes lies in the extreme RNA replication occurring in the cytoplasm of host cells, which will improve the efficacy and also reduce the dose of DNA required to obtain a strong immune response. Moreover, the replicons induce interferon and perhaps other cytokines, which stimulate the immune system.

Alphaviruses have demonstrated their usefulness as carriers for vaccines against a broad range of viral, bacterial and parasitic pathogens as well as tumor cells (40). Although most of these studies were performed in the murine model, the efficacy of these vectors has also been demonstrated in a number of other animal models such as hamster, guinea-pig, chicken, sheep, and macaque. One attraction of the VEE replicon particle 
is the ability of VEE glycoprotein E2 to target the virus and replicon particles to dendritic cells. This can be an important step for antigen presentation to T-cells and could serve as a stimulus for the induction of humoral and cell-mediated immune responses. It has been shown that in mice the resident dendritic cells in the skin (Langerhans cells) are the first cells infected by VEE replicon particles (41). These cells are activated and move to the draining lymph nodes. However, this property is not unique to VEE particles. Recently, it was shown that a single mutation in the E2 glycoprotein of SIN virus could preferentially target virus infection to human dendritic cells. In this regard, packaging of SIN replicon particles with the mutant E2 glycoprotein resulted in enhanced infection of human dendritic cells in vitro (42). These studies further demonstrate the importance of having suitable cell tropism, conferred by the glycoprotein coat of the infecting alphavirus replicon particles, to elicit the desired immune response. The first clinical trial of an alphavirus-based replicon vaccine employing a subtype $\mathrm{c}$ HIV gag gene expressed by a VEE replication particle was scheduled to begin a few years ago and this study will undoubtedly help determine the usefulness of alphaviruses as vaccine carriers.

\section{Poliovirus}

Poliovirus is a member of the Picornaviridae family of positive-stranded RNA viruses with a genome of approximately 7400 nucleotides. The mature poliovirus has a non-enveloped icosahedral protein shell that is composed of four structural proteins: VP1, VP2, VP3, and VP4 (43). Poliovirus is the etiological agent of poliomyelitis, and thus all poliovirus vectors have been derived from the well-characterized and attenuated Sabin vaccine strains. This is particularly appealing due to their widely accepted safety profiles in billions of vaccinees, as well as the extensively characterized ability of these vaccines to induce strong long-term immune responses in humans. The use of picornavirus as a vaccine vector has many other benefits: it is easy to deliver orally without the use of needles, and it is inherently stable in the intestinal tract where it infects the mucosal $\mathrm{M}$ cells responsible for antigenic presentation, inducing a potent mucosal immune response (44). Manufacturing procedures for poliovirus are well established and the virus can be grown to high titers, enabling low cost production of this viral vaccine vector.

To date, two approaches have been used to develop vaccines based upon poliovirus, i.e., replication competent and replication defective. In the first approach, the capsid proteins are modified to include immunogenic peptides from other microorganisms within the virion capsid protein. The heterologous sequence is flanked by protease cleavage sites. Thus, all the poliovirus protein components are produced normally and viral replication is not compromised. This method yields recombinant viruses that are capable of replicating and propagating within a host without the need for a helper virus (45). However, the amount of new genetic material that can be introduced using this approach is severely limited. In the second methodology poliovirus vectors are constructed as replicons in which the virion coat protein genes VP2 and VP3 are replaced "inframe" with a heterologous gene. The generation of poliovirus replicons is possible due to the fact that the virus P1 gene, which encodes the capsid proteins, is not essential for RNA replication and this region can be replaced with foreign material (46). The release of the expressed gene can be achieved by flanking the insert with engineered viral protease cleavage sites. Replicon RNA is packaged into virus-like particles by transfecting in vitro transcribed RNA into cells infected with helper poliovirus or with a vaccinia virus recombinant expressing the intact poliovirus P1 region (46). 
Both replication-competent and replication-deficient recombinant polioviruses have been shown to be immunogenic in several experimental systems such as studies in nonhuman primates and using various routes of immunization, including mucosal delivery (47). Although polioviruses do offer a potentially powerful vaccine delivery system, there are two significant restrictions of the use of live picornavirus vectors: the stability and size (which is approximately 200 amino acids) of heterologous gene inserts (48) and the presence of high levels of preexisting immunity to polio vectors in the general population.

\section{Herpes viruses}

Herpes simplex virus 1 (HSV-1), a major cause of sexually transmitted disease, is an enveloped virus containing a double-stranded DNA genome of $152 \mathrm{~kb}$, which replicates in the nucleus. HSV is a suitable candidate vaccine vector as a substantial number of viral genes are considered non-essential for virus growth in cell culture and consequently large or multiple transgenes can be inserted into the HSV genome without affecting its ability to replicate (49). Various aspects of the biology of HSV-1 make it attractive as a vaccine vector: broad host cell range, high infectivity, ability to transduce non-dividing cells, and the ease of production of hightiter, high-purity stocks of virus. Another important advantage of HSV-based vectors is the facilitation of long-term expression of foreign antigens since herpesvirus exhibits life-long persistence in the host along with an HSV-specific immune response.

Despite the fact that initial herpes vectors were replication competent, current efforts focus on the development of replicationincompetent viruses $(50,51)$. In one approach, deletion of nine HSV-1 immediate early genes substantially reduced vector toxicity while allowing for the expression of multiple transgenes (50). In another approach, disabled, infectious, single-cycle herpes simplex vectors were engineered both as vaccines against HSV and as vectors for cancer immunotherapy (52). The genetically disabled vector lacks the gene for the essential glycoprotein $\mathrm{h}(\mathrm{gH})$ and has been proven to have a good safety profile (51). Generally, the disabled vector is manufactured by propagating the virus in a complementing cell line producing the gH glycoprotein. Recombinants are created by recombination of the HSV genome and a transfected plasmid carrying the gene of interest flanked by HSV-1 sequences.

Several herpes virus recombinants have been used against a range of veterinary as well as human diseases $(53,54)$. Recombinant herpes virus offers many advantages to a vaccinologist. The viral genome is easy to manipulate and is genetically stable. The insertion of large amounts of foreign genes could be used to construct multivalent vaccines. Notably, HSV stimulates humoral and cellular immune responses. Nevertheless, the overall toxicity of herpes viruses presents a significant drawback to their widespread use as vaccine vectors. HSV-1 is neurotoxic and its virion proteins shut off cell protein synthesis. Additionally, the issue of pre-existing immunity against $\mathrm{HSV}$ in the human population also needs to be addressed in order to use this vector. So far it has not been possible to produce a virus that replicates well, is highly immunogenic and safe for use in humans.

\section{Prime-boost regimen}

Recently, prime-boost strategies have increased in popularity due to their ability to generate stronger immune responses compared to regimes using a single vaccination. In general, viral vectors are very good at boosting DNA-primed immune responses. This effect was first demonstrated in a model using the hemagglutinin gene of influenza virus in a DNA/rFPV regime, in which a Th1 response was developed and protection was 
achieved in mice (55). An immunization strategy that included priming with plasmid DNA followed by boosting with Ad vector has been shown to augment immune responses compared with plasmid DNA alone $(23,24)$. Also, the combination of DNA/MVA controlled a highly pathogenic immunodeficiency virus challenge in a rhesus macaque model (56) and partial protection has been reported in humans against a challenge with P. falciparum (12). Moreover, with the availability of more engineered viral vectors, the prime-boost strategies have been benefited. Combinations involving DNA, MVA, and adenovirus of the human serotype 5 have shown great potential. Both viral vectors can boost a DNA-primed response, and in a similar way rAdH5 can prime or boost an rMVA response resulting in an even greater response. However, when protection is analyzed, the best combination has consisted of rAdH5 priming followed by an rMVA boost (57). An interesting study in which a recombinant vaccinia virus boosted an rAd-prime response to the CS protein of $P$. yoelii showed clear evidence that timing between prime and boost can be critical to achieve complete protection, and further characterization of the immune response elicited by the diverse viral vectors should be carefully studied in order to maximize their effects (58). On the other hand, immunity to the viral vectors is also generated and this will play an important role limiting the subsequent use of the same platform for different vaccination purposes. In the case of MVA, consecutive administration does not increase significantly the levels of immunogenicity, and pre-existing immunity to MVA reduces the effect of subsequent priming or boosting with the same vector (59). In this regard, it would be of interest to analyze the effect of the preexisting immunity during long periods of time between vaccinations, as well as a variation in the doses for priming or boosting. Similar effects are seen with rAd, in which a homologous boost does not improve the im- mune response elicited by a single rAd prime, whereas a heterologous $\mathrm{rAd} / \mathrm{rMVA}$ primeboost can significantly increase anti-HIV-1 cell-mediated immunity in rhesus macaques (60). It is of interest that the same vectors do not elicit a good immune response when administered in the reverse order, and in this system poxviruses can be used consecutively as boosters to maintain the cellular immunity. Good cellular and humoral immunity has also been achieved by heterologous primeboost with different serotypes of adenovirus in rhesus macaques (30), and the generation of new serotypes of adenoviral vectors originated from chimpanzees will increase the potential combinations for prime-boost regimes and could allow to overcome problems such as pre-existing immunity (28).

\section{Perspectives}

Viral vectors offer important advantages when used as vaccines and they have unique characteristics as shown in Table 1. These vectors facilitate the production of high levels of proteins inside the cells that are transduced. Among the cells that can be transduced by these vectors, antigen-presenting cells constitute an important subset that can be targeted by different viral vectors, making the transgenic protein accessible to the immune system. Also, the vector itself can provide an adjuvant effect enhancing the immune response against the transgenic protein. Although a single vector should not be seen as a universal vaccine carrier appropriate for use in vaccine development against every disease, the characteristics of each virus vector-based vaccine can be exploited for use in specific cases. Moreover, the feasibility of administering viral vector-derived vaccines in combination modalities with other vaccines gives these vaccines an additional advantage. From this review, it is clear that viral vectors are gaining an important place in the field of vaccinology. For many years, traditional vaccines have proved to be 
successful in the prevention and eradication of numerous infectious diseases. The approaches used to develop vaccines were initially empirical and were dependent upon the induction of neutralizing antibodies to generate protection. Nevertheless, for some emerging diseases this approach may not be successful mainly due to the need to generate a broad spectrum of immune responses including neutralizing antibodies, T-cell responses as well as mucosal immunity that has been shown to correlate with protection. For diseases that require a more complex immune response, such as HIV and malaria, viral vectors may constitute the most important hope for the development of a successful vaccine.

\section{Acknowledgments}

The authors would like to thank Dr. Carlos Roberto Zanetti, Departamento de Microbiologia e Parasitologia, UFSC, Florianópolis, SC, Brazil, and Dr. Thiru Vanniasinkam, The Wistar Institute, Philadelphia, PA, USA, for critically reading the manuscript.

\begin{tabular}{|c|c|c|c|c|c|c|c|c|}
\hline Vector & Biology & $\begin{array}{l}\text { Preexisting immunity } \\
\text { against vector }\end{array}$ & $\begin{array}{l}\text { Main immune } \\
\text { response }\end{array}$ & $\begin{array}{l}\text { Infection } \\
\text { of APC }\end{array}$ & $\begin{array}{l}\text { Duration of } \\
\text { gene } \\
\text { expression }\end{array}$ & Safety profile & $\begin{array}{c}\text { Size of } \\
\text { insert }\end{array}$ & Ref. \\
\hline $\begin{array}{l}\text { Vaccinia and } \\
\text { highly attenuated } \\
\text { vaccinia strains }\end{array}$ & $\begin{array}{l}\text { Double-stranded DNA } \\
\text { virus replication } \\
\text { competent (attenuated) }\end{array}$ & $\begin{array}{l}\text { Yes (in smallpox } \\
\text { vaccinees) }\end{array}$ & $\mathrm{CTL}, \mathrm{CD} 4$ & Yes & $\begin{array}{l}\text { Transient } \\
\text { (days) }\end{array}$ & $\begin{array}{l}\text { Safe, except in } \\
\text { immunosuppressed } \\
\text { hosts. Safety well } \\
\text { documented }\end{array}$ & $\begin{array}{l}30 \mathrm{~kb} \\
\text { or more }\end{array}$ & $\begin{array}{r}11 \\
12 \\
56\end{array}$ \\
\hline $\begin{array}{l}\text { Avipox viruses } \\
\text { (Canarypox, } \\
\text { Fowlpox) }\end{array}$ & $\begin{array}{l}\text { Double-stranded DNA } \\
\text { virus that does not } \\
\text { replicate in mammalian } \\
\text { cells }\end{array}$ & No & CTL, CD4 & Yes & $\begin{array}{l}\text { Transient } \\
\text { (days) }\end{array}$ & Very safe & $\begin{array}{l}30 \mathrm{~kb} \\
\text { or more }\end{array}$ & $\begin{array}{l}11 \\
14 \\
15\end{array}$ \\
\hline Adenovirus & $\begin{array}{c}\text { Double-stranded DNA } \\
\text { virus }\end{array}$ & $\begin{array}{l}\text { Yes (highly } \\
\text { immunogenic) }\end{array}$ & $\mathrm{Ab}, \mathrm{CTL}, \mathrm{CD} 4$ & Yes & $\begin{array}{l}\text { Transient } \\
\text { (days) }\end{array}$ & Safe & $8-9$ kb & $\begin{array}{l}23 \\
24 \\
28\end{array}$ \\
\hline $\begin{array}{l}\text { Alphaviruses } \\
\text { (Semliki Forest } \\
\text { virus, equine } \\
\text { encephalitis virus) }\end{array}$ & RNA virus with replicon & No & $\mathrm{Ab}, \mathrm{CTL}$ & Yes & Good & $\begin{array}{l}\text { Not tested in } \\
\text { humans, safe in } \\
\text { animals }\end{array}$ & $\begin{array}{l}\text { Up to } \\
8 \mathrm{~kb}\end{array}$ & $\begin{array}{l}34 \\
38 \\
40\end{array}$ \\
\hline $\begin{array}{l}\text { Herpes simplex } \\
\text { virus }\end{array}$ & $\begin{array}{c}\text { Double-stranded DNA } \\
\text { virus }\end{array}$ & Yes & $\mathrm{Ab}$ & Yes & Transient & $\begin{array}{l}\text { Associated with } \\
\text { neurovirulence, } \\
\text { insertional } \\
\text { mutagenesis }\end{array}$ & $30 \mathrm{~kb}$ & $\begin{array}{l}52- \\
54\end{array}$ \\
\hline Poliovirus & Single-stranded RNA & Yes & $A b, C T L$ & Yes & Transient & $\begin{array}{l}\text { Safe (derived from } \\
\text { the attenuated } \\
\text { Sabin strain) }\end{array}$ & $<1 \mathrm{~kb}$ & $\begin{array}{l}45- \\
47\end{array}$ \\
\hline
\end{tabular}

$\mathrm{APC}=$ antigen presenting cells; $\mathrm{CTL}=$ cytolytic $\mathrm{T}$ lymphocytes $; \mathrm{CD} 4=$ helper $\mathrm{T}$ lymphocytes; $\mathrm{Ab}=$ antibodies 


\section{References}

1. Reyes-Sandoval A \& Ertl HC (2001). DNA vaccines. Current Molecular Medicine, 1: 217-243.

2. Panicali D, Davis SW, Weinberg RL \& Paoletti E (1983). Construction of live vaccines by using genetically engineered poxviruses: biological activity of recombinant vaccinia virus expressing influenza virus hemagglutinin. Proceedings of the National Academy of Sciences, USA, 80: 5364-5368.

3. Goebel SJ, Johnson GP, Perkus ME, Davis SW, Winslow JP \& Paoletti E (1990). The complete DNA sequence of vaccinia virus. Virology, 179: 247-266, 517-563.

4. Robinson HL (2002). New hope for an AIDS vaccine. Nature Reviews. Immunology, 2: 239-250.

5. Perkus ME, Piccini A, Lipinskas BR \& Paoletti E (1985). Recombinant vaccinia virus: immunization against multiple pathogens. Science, 229: 981-984.

6. Mackett M, Smith GL \& Moss B (1984). General method for production and selection of infectious vaccinia virus recombinants expressing foreign genes. Journal of Virology, 49: 857-864.

7. Merchlinsky M \& Moss $B$ (1992). Introduction of foreign DNA into the vaccinia virus genome by in vitro ligation: recombination-independent selectable cloning vectors. Virology, 190: 522-526.

8. Pastoret PP \& Brochier B (1996). The development and use of a vaccinia-rabies recombinant oral vaccine for the control of wildlife rabies; a link between Jenner and Pasteur. Epidemiology and Infection, 116: 235-240.

9. Sutter G \& Moss B (1992). Nonreplicating vaccinia vector efficiently expresses recombinant genes. Proceedings of the National Academy of Sciences, USA, 89: 10847-10851.

10. Tartaglia J, Perkus ME, Taylor J et al. (1992). NYVAC: a highly attenuated strain of vaccinia virus. Virology, 188: 217-232.

11. Kanesa-Thasan N, Smucny JJ, Hoke CH, Marks DH, Konishi E, Kurane I, Tang DB, Vaughn DW, Mason PW \& Shope RE (2000). Safety and immunogenicity of NYVAC-JEV and ALVAC-JEV attenuated recombinant Japanese encephalitis virus-poxvirus vaccines in vaccinia-nonimmune and vaccinia-immune humans. Vaccine, 19: 483-491.

12. McConkey SJ, Reece WH, Moorthy VS et al. (2003). Enhanced Tcell immunogenicity of plasmid DNA vaccines boosted by recombinant modified vaccinia virus Ankara in humans. Nature Medicine, 9: 729-735.

13. Paoletti E (1996). Applications of pox virus vectors to vaccination: an update. Proceedings of the National Academy of Sciences, USA, 93: 11349-11353.

14. Taylor J, Weinberg R, Languet B, Desmettre P \& Paoletti E (1988). Recombinant fowlpox virus inducing protective immunity in nonavian species. Vaccine, 6: 497-503.

15. Konishi E, Kurane I, Mason PW, Shope RE, Kanesa-Thasan N, Smucny JJ, Hoke Jr CH \& Ennis FA (1998). Induction of Japanese encephalitis virus-specific cytotoxic $T$ lymphocytes in humans by poxvirus-based JE vaccine candidates. Vaccine, 16: 842-849.

16. Top Jr FH, Grossman RA, Bartelloni PJ, Segal HE, Dudding BA, Russell PK \& Buescher EL (1971). Immunization with live types 7 and 4 adenovirus vaccines. I. Safety, infectivity, antigenicity, and potency of adenovirus type 7 vaccine in humans. Journal of Infectious Diseases, 124: 148-154.

17. Pinto AR \& Ertl HC (2002). Genetically modified adenoviruses as recombinant vaccines. Current Topics in Virology, 2: 70-84.

18. Graham FL \& Prevec L (1992). Adenovirus-based expression vec- tors and recombinant vaccines. Biotechnology, 20: 363-390.

19. Berkner KL (1988). Development of adenovirus vectors for the expression of heterologous genes. BioTechniques, 6: 616-629.

20. Graham FL, Smiley J, Russell WC \& Nairn R (1977). Characteristics of a human cell line transformed by DNA from human adenovirus type 5. Journal of General Virology, 36: 59-74.

21. Ng P, Parks RJ, Cummings DT, Evelegh CM \& Graham FL (2000). An enhanced system for construction of adenoviral vectors by the two-plasmid rescue method. Human Gene Therapy, 11: 693-699.

22. Fallaux FJ, Bout A, van der Velde I et al. (1998). New helper cells and matched early region 1-deleted adenovirus vectors prevent generation of replication-competent adenoviruses. Human Gene Therapy, 9: 1909-1917.

23. Sullivan NJ, Sanchez A, Rollin PE, Yang ZY \& Nabel GJ (2000). Development of a preventive vaccine for Ebola virus infection in primates. Nature, 408: 605-609.

24. Shiver JW, Fu TM, Chen L et al. (2002). Replication-incompetent adenoviral vaccine vector elicits effective anti-immunodeficiencyvirus immunity. Nature, 415: 331-335.

25. Farina SF, Gao GP, Xiang ZQ, Rux JJ, Burnett RM, Alvira MR, Marsh J, Ertl HC \& Wilson JM (2001). Replication-defective vector based on a chimpanzee adenovirus. Journal of Virology, 75: 11603-11613.

26. Gahery-Segard H, Molinier-Frenkel V, Le Boulaire C et al. (1997). Phase I trial of recombinant adenovirus gene transfer in lung cancer. Longitudinal study of the immune responses to transgene and viral products. Journal of Clinical Investigation, 100: 2218-2226.

27. Papp Z, Babiuk LA \& Baca-Estrada ME (1999). The effect of preexisting adenovirus-specific immunity on immune responses induced by recombinant adenovirus expressing glycoprotein $D$ of bovine herpesvirus type 1. Vaccine, 17: 933-943.

28. Pinto AR, Fitzgerald JC, Giles-Davis W, Gao GP, Wilson JM \& Ertl HC (2003). Induction of CD8+ T cells to an HIV-1 antigen through a prime boost regimen with heterologous E1-deleted adenoviral vaccine carriers. Journal of Immunology, 171: 6774-6779.

29. Pinto AR, Fitzgerald JC, Gao GP, Wilson JM \& Ertl HC (2004). Induction of CD8+ T cells to an HIV-1 antigen upon oral immunization of mice with a simian E1-deleted adenoviral vector. Vaccine, 22: 697-703.

30. Reyes-Sandoval A, Fitzgerald JC, Grant R, Roy S, Xiang ZQ, Li Y, Gao GP, Wilson JM \& Ertl HC (2004). Human immunodeficiency virus type 1-specific immune responses in primates upon sequential immunization with adenoviral vaccine carriers of human and simian serotypes. Journal of Virology, 78: 7392-7399.

31. Strauss JH \& Strauss EG (1994). The alphaviruses: gene expression, replication, and evolution. Microbiological Reviews, 58: 491562.

32. Schlesinger S \& Dubensky TW (1999). Alphavirus vectors for gene expression and vaccines. Current Opinion in Biotechnology, 10: 434-439.

33. Albert ML, Sauter B \& Bhardwaj N (1998). Dendritic cells acquire antigen from apoptotic cells and induce class I-restricted CTLs. Nature, 392: 86-89.

34. Bennett AM, Elvin SJ, Wright AJ, Jones SM \& Phillpotts RJ (2000). An immunological profile of Balb/c mice protected from airborne challenge following vaccination with a live attenuated Venezuelan equine encephalitis virus vaccine. Vaccine, 19: 337-347.

35. Liljestrom P \& Garoff H (1991). A new generation of animal cell expression vectors based on the Semliki Forest virus replicon. 
Biotechnology, 9: 1356-1361.

36. Frolov I, Frolova E \& Schlesinger S (1997). Sindbis virus replicons and Sindbis virus: assembly of chimeras and of particles deficient in virus RNA. Journal of Virology, 71: 2819-2829.

37. Polo JM, Belli BA, Driver DA et al. (1999). Stable alphavirus packaging cell lines for Sindbis virus and Semliki Forest virus-derived vectors. Proceedings of the National Academy of Sciences, USA, 96: 4598-4603.

38. Zhou X, Berglund P, Rhodes G, Parker SE, Jondal M \& Liljestrom P (1994). Self-replicating Semliki Forest virus RNA as recombinant vaccine. Vaccine, 12: 1510-1514.

39. Herweijer H, Latendresse JS, Williams P, Zhang G, Danko I, Schlesinger S \& Wolff JA (1995). A plasmid-based self-amplifying Sindbis virus vector. Human Gene Therapy, 6: 1161-1167.

40. Lundstrom K (2003). Alphavirus vectors for vaccine production and gene therapy. Expert Review of Vaccines, 2: 447-459.

41. MacDonald GH \& Johnston RE (2000). Role of dendritic cell targeting in Venezuelan equine encephalitis virus pathogenesis. Journal of Virology, 74: 914-922.

42. Gardner JP, Frolov I, Perri S et al. (2000). Infection of human dendritic cells by a Sindbis virus replicon vector is determined by a single amino acid substitution in the E2 glycoprotein. Journal of Virology, 74: 11849-11857.

43. Pallansch MA, Kew OM, Semler BL, Omilianowski DR, Anderson CW, Wimmer E \& Rueckert RR (1984). Protein processing map of poliovirus. Journal of Virology, 49: 873-880.

44. Ogra PL \& Ogra SS (1973). Local antibody response to poliovaccine in the human female genital tract. Journal of Immunology, 110: 1307-1311.

45. Andino R, Silvera D, Suggett SD, Achacoso PL, Miller CJ, Baltimore $D$ \& Feinberg MB (1994). Engineering poliovirus as a vaccine vector for the expression of diverse antigens. Science, 265: 1448-1451.

46. Porter DC, Ansardi DC, Choi WS \& Morrow CD (1993). Encapsidation of genetically engineered poliovirus minireplicons which express human immunodeficiency virus type $1 \mathrm{Gag}$ and Pol proteins upon infection. Journal of Virology, 67: 3712-3719.

47. Crotty S, Miller CJ, Lohman BL, Neagu MR, Compton L, Lu D, Lu FX, Fritts L, Lifson JD \& Andino R (2001). Protection against simian immunodeficiency virus vaginal challenge by using Sabin poliovirus vectors. Journal of Virology, 75: 7435-7452.

48. Mueller S \& Wimmer E (1998). Expression of foreign proteins by poliovirus polyprotein fusion: analysis of genetic stability reveals rapid deletions and formation of cardioviruslike open reading frames. Journal of Virology, 72: 20-31.

49. Burton EA, Wechuck JB, Wendell SK, Goins WF, Fink DJ \& Glorioso JC (2001). Multiple applications for replication-defective herpes simplex virus vectors. Stem Cells, 19: 358-377.

50. Krisky DM, Marconi PC, Oligino TJ, Rouse RJ, Fink DJ, Cohen JB,
Watkins SC \& Glorioso JC (1998). Development of herpes simplex virus replication-defective multigene vectors for combination gene therapy applications. Gene Therapy, 5: 1517-1530.

51. McLean CS, Erturk M, Jennings $R$, Challanain DN, Minson AC, Duncan I, Boursnell ME \& Inglis SC (1994). Protective vaccination against primary and recurrent disease caused by herpes simplex virus (HSV) type 2 using a genetically disabled HSV-1. Journal of Infectious Diseases, 170: 1100-1109.

52. Todryk S, McLean C, Ali S, Entwistle C, Boursnell M, Rees R \& Vile $R$ (1999). Disabled infectious single-cycle herpes simplex virus as an oncolytic vector for immunotherapy of colorectal cancer. Human Gene Therapy, 10: 2757-2768.

53. Murphy CG, Lucas WT, Means RE, Czajak S, Hale CL, Lifson JD, Kaur A, Johnson RP, Knipe DM \& Desrosiers RC (2000). Vaccine protection against simian immunodeficiency virus by recombinant strains of herpes simplex virus. Journal of Virology, 74: 7745-7754.

54. Morgan RW, Gelb Jr J, Schreurs CS, Lutticken D, Rosenberger JK \& Sondermeijer PJ (1992). Protection of chickens from Newcastle and Marek's diseases with a recombinant herpesvirus of turkeys vaccine expressing the Newcastle disease virus fusion protein. Avian Diseases, 36: 858-870.

55. Leong KH, Ramsay AJ, Morin MJ, Robinson HL, Boyle DB \& Ramshaw IA (1995). Generation of enhanced immune responses by consecutive immunization with DNA and recombinant fowlpox viruses. In: Brown F, Chanock H \& Norrby E (Editors), Vaccines 95. Cold Spring Harbor Laboratory Press, Cold Spring Harbor, NY, USA.

56. Amara RR, Villinger F, Altman JD et al. (2001). Control of a mucosal challenge and prevention of AIDS by a multiprotein DNA/MVA vaccine. Science, 292: 69-74.

57. Gilbert SC, Schneider J, Hannan CM, Hu JT, Plebanski M, Sinden R \& Hill AV (2002). Enhanced CD8 T cell immunogenicity and protective efficacy in a mouse malaria model using a recombinant adenoviral vaccine in heterologous prime-boost immunisation regimes. Vaccine, 20: 1039-1045.

58. Bruna-Romero O, Gonzalez-Aseguinolaza G, Hafalla JC, Tsuji M \& Nussenzweig RS (2001). Complete, long-lasting protection against malaria of mice primed and boosted with two distinct viral vectors expressing the same plasmodial antigen. Proceedings of the $\mathrm{Na}$ tional Academy of Sciences, USA, 98: 11491-11496.

59. Hanke T, Blanchard TJ, Schneider J, Hannan CM, Becker M, Gilbert SC, Hill AV, Smith GL \& McMichael A (1998). Enhancement of MHC class I-restricted peptide-specific T cell induction by a DNA prime/ MVA boost vaccination regime. Vaccine, 16: 439-445.

60. Casimiro DR, Bett AJ, Fu TM et al. (2004). Heterologous human immunodeficiency virus type 1 priming-boosting immunization strategies involving replication-defective adenovirus and poxvirus vaccine vectors. Journal of Virology, 78: 11434-11438. 